\title{
MOBILIDADE FÍSICA PREJUDICADA EM IDOSOS: FATORES RELACIONADOS E CARACTERÍSTICAS DEFINIDORAS
}

\author{
IMPAIRED PHYSICAL MOBILITY AMONG ELDER PEOPLE: RELATED FACTORS AND \\ DEFINING CHARACTERISTICS
}

\author{
MOBILIDAD FÍSICA PERJUDICADA EN ANCIANOS: FACTORES RELACIONADOS Y \\ CARACTERÍSTICAS DEFINIDORAS
}

Lorena Aparecida de Oliveira Araújo ${ }^{1}$ Rosimere Ferreira Santana ${ }^{2}$ Maria Márcia Bachion ${ }^{3}$

RESUMO: Este estudo exploratório objetivou levantar a ocorrência do Diagnóstico de Enfermagem "Mobilidade física prejudicada" em idosos institucionalizados. Foi desenvolvido em uma instituição asilar em Goiânia (GO). Para coleta de dados foi utilizado um protocolo que incluiu entrevista, observação, exame físico e consulta à membros da equipe que prestava assistência ao idoso. Participaram 60 idosos, com idade entre 60 a 105 anos. Foi identificado "Mobilidade física prejudicada" em $100 \%$ dos sujeitos, geralmente relacionada ao enfraquecimento músculo-esquelético $(76,7 \%)$, força e resistência muscular diminuídas $(61,7 \%)$ e enfraquecimento neuro-muscular $(55 \%)$ além de dano perceptual ou cognitivo $(53,3 \%)$. As caracteristicas definidoras mais freqüentes foram: flexibilidade diminuida $(96,7 \%)$, comprometimento das articulações $(91,7 \%)$, inabilidade para mover-se significativamente dentro do ambiente físico $(83,63 \%)$, dentre outros.

PALAVRAS-CHAVE: diagnóstico de enfermagem, idosos, mobilidade fisica prejudicada

ABSTRACT: The present exploratory study aims at estimating the occurrence of the Nursing Diagnosis "Impaired Physical Mobility' in elderly who live in a home institution in the city of Goiânia, Goiás. The study was carried out from December 1999 to February 2000. The collection of data was done through interviews, observations, physical exam and information obtained from the professionals who assisted the elderly. Sixty patients ranging from 60 to 105 years were the subjects of this study. Results showed $100 \%$ of the elderly were impaired regarding physical mobility. This impairment was generally related to muscular-skeletal- weakness $(76,7 \%)$, reduced muscle resistance and strength $(61,7 \%)$, neuro-muscular weakness (55\%), besides perceptional or cognitive damage $(53,3 \%)$. The most frequent defining characteristics were: decreased flexibility $(96,7 \%)$, jeopardized articulations (96,7\%), and inability to move in a physical environment $(83,3 \%)$.

KEYWORDS: elderly people, impaired physical mobility, nursing diagnosis

RESUMEN: Desarrollamos este estudio exploratorio para levantar las ocurrencias del Diagnóstico de Enfermeria "Mobilidad física perjudicada" en los ancianos institucionalizados. El estudio fue desarrollado desde diciembre de 1999 hasta febrero de 2000 en un asilo de Goiânia-GO. El levantamiento de datos se hizo a través del protocolo de la institución, que incluye entrevista, observación, examen fisico y consulta a los miembros del grupo que da asistencia al anciano. Obtuvimos la participación de 60 ancianos con edades que oscilan entre los 60 y los 105 años, aunque la mayoría esté entre 60 y 69 años. Verificanos que $100 \%$ de las personas presentan mobilidad fisica perjudicada, generalmente relacionada al adelgazamiento del músculo esquelético $(76,7 \%)$, fuerza y resistencia muscular disminuida $(61,7 \%)$ y adelgazamiento neuro-muscular $(55 \%)$, además del daño perceptual o cognitivo (53,3\%). Las manifestaciones más frecuentes son inhabilidad para moverse significativamente dentro del ambiente físico $(83,63 \%)$, flexibilidad disminuida $(96,7 \%)$, articulaciones comprometidas $(91,7 \%)$, entre otros. PALABRAS CLAVE: ancianos, diagnóstico de enfermeria, mobilidad fisica perjudicada

Recebido em 17/11/2001

Aprovado em 06/02/2002

\footnotetext{
${ }^{1}$ Enfermeira do Programa Saúde da Familia em Goiânia. Ex-Bolsista Iniciação Científica PIBIC/UFG/CNPq.

${ }^{2}$ Enfermeira. Aluna do Curso de Especialização em Psicogeriatria, no Centro de Doença de Alzheimer do instituto de Psiquiatria da Universidade Federal do Rio de Janeiro.

${ }^{3}$ Doutor em Enfermagem. Professor Titular da Faculdade de Enfermagem da Universidade Federal de Goiás.
} 


\section{INTRODUÇÃO}

A mobilidade é essencial para a manutenção de uma vida independente. Além disso, interfere em vários aspectos tais como: o padrão de funcionamento intestinal, o estado nutricional, o auto conceito, a auto imagem, dentre outros. A diminuição da mobilidade física predispõe às infecções do sistema respiratório e urinário (CARPENITO, 1997; DOENGES; MOORHOUSE, 1999).

A população idosa atingiu $8 \%$ da população total em 1992, o que equivale a aproximadamente 13 milhões de individuos com idade acima de 60 anos. A projeção deste número para 2001 é de 14 milhões e para 2025 de 32 milhões (PAPALÉO NETO, 2000).

É necessário que a Enfermagem busque preparo adequado para o atendimento destas pessoas. Uma das formas de organizar o cuidar em enfermagem é o Processo de Enfermagem, que inclui as fases: coleta de dados, diagnóstico de enfermagem, planejamento da assistência de enfermagem, intervenções de enfermagem e avaliação de enfermagem. Estas etapas são inter-relacionadas e interdependentes.

A coleta de dados envolve o levantamento de informações multifocais sobre o cliente, através da entrevista, observação, exame físico e consulta aos registros e resultados de exames. Mediante raciocínio clinico, a partir dos dados coletados, o enfermeiro realiza o julgamento clínico, identificando diagnósticos de enfermagem, que podem ser genericamente definidos como condições que requerem assistência de enfermagem e que direcionarão o planejamento das ações necessárias para atingir os resultados pêlos quais a enfermagem é responsável.

Atualmente existem vários sistemas de classificação (taxonomias) de diagnósticos de enfermagem. Dentre estas, uma das mais conhecidas é a Taxonomia I Revisada da North American Nursing Diagnosis Association (NANDA, 1994, 2000), que adotamos no presente estudo.

A Mobilidade Física Prejudicada é um Diagnóstico de Enfermagem aceito na classificação da NANDA em 1973, cuja definição permaneceu até 1996 como: "estado no qual o individuo experimenta uma limitação na habilidade para movimentos físicos independentes (NANDA, 1994, p.60). Na revisão de 1998, esta passou a ser entendida como "uma limitação no movimento físico independente voluntário do corpo ou de uma ou mais extremidades" (NANDA, 2000, p. 87).

No decorrer do processo de envelhecimento a pessoa sofre modificações significativas na área motora (PAPALÉO NETO, 2000, SMELTZER; BARE, 1998, OKUMA, 1998, RODRIGUES; DIOGO, 1996, MORAIS; BRAGA; SILVA, 1995), o que nos leva a acreditar que a Mobilidade Fisica Prejudicada tenha alta ocorrência em idosos.

Diante do exposto, optamos por realizar esta pesquisa com o objetivo de fazer um levantamento da ocorrência do diagnóstico de enfermagem "Mobilidade Fisica Prejudicada" em idosos institucionalizados.

\section{MÉTODO}

Trata-se de um estudo quantitativo do tipo exploratório, realizado no Abrigo Sagrada Família, em Goiânia (GO), que é uma instituição Filantrópica, sem fins lucrativos, coordenada pela Organização das Voluntárias do Estado de Goiás (O.V.G.), que recebe idosos que não conseguem realizar atividade independentemente e necessitam de cuidados de saúde. A coleta de dados ocorreu no mês de Dezembro de 1999, Janeiro e Fevereiro de 2000 com os idosos moradores da instituição.

Após aquiescência da O.V.G., foram abordados os 75 moradores do local no período de coleta de dados. Sessenta individuos, após esclarecidos os objetivos e metodologia da pesquisa, concordaram em participar e nove se recusaram. Seis possuiam menos de 60 anos e foram excluidos da amostra.

Para a coleta dos dados aplicou-se um protocolo previamente construido e utilizado por Araújo e Bachion (1999) e Bachion et al. (1999). O procedimento incluiu entrevista, observação, exame físico e consulta a diferentes membros da equipe de saúde local, assim como no prontuário do idoso.

\section{RESULTADO E DISCUSSÃO}

Dos sessenta sujeitos que participaram, 32 são do sexo feminino e 28 do sexo masculino. A faixa etária variou de 60 a 105 anos, sendo predominante a que está no intervalo entre 60 e 69 anos, na qual estão 31 indivíduos, seguida pela faixa etária entre 70 e 79 anos com 12 individuos. Na faixa etária que vai dos 80 até 89 anos obtivemos 10 idosos e na faixa entre 90 e 99 anos foram encontrados 06 idosos. Com mais de 100 anos participou um individuo, que apresentava 105 anos.

Após a aplicação do protocolo pelos pesquisadores pudemos identificar "Mobilidade Física Prejudicada" em 100\% dos sujeitos. Segundo Brady e Foley citado por Carpenito (1997) acima de 90\% dos idosos institucionalizados são dependentes em, no mínimo, uma atividade de vida diária, e estes problemas referentes à mobilidade são causa para a admissão destes indivíduos às casas geriátricas ou ao tratamento intensivo em casa.

Com relação aos Fatores Relacionados, o "Enfraquecimento músculo esquelético" foi o que 
apareceu em maior freqüência, estando presente em 46 sujeitos (Tabela 1 ).

Segundo a literatura, este é uns dos problemas mais comuns que levam à diminuição da mobilidade (SMELTZER; BARE, 1998).

TABELA 1 - Fatores Relacionados do Diagnóstico de Enfermagem Mobilidade Fisica Prejudicada em idosos Goiânia - 2000

\begin{tabular}{lcc}
\hline \multicolumn{1}{c}{ Fatores Relacionados } & $F$ & $\%$ \\
\hline Enfraquecimento Músculo-esquelético & 46 & 76,7 \\
Força e Resistência Diminuídas & 37 & 61,7 \\
Enfraquecimento Neuromuscular & 33 & 55,0 \\
Dano Perceptual ou Cognitivo & 32 & 53,3 \\
Dor ou Desconforto & 22 & 36,7 \\
Intolerância à Atividade Fisica & 17 & 28,3 \\
Depressão ou Ansiedade Severa & 09 & 15,0
\end{tabular}

Vários autores (PAPALÉO NETO, 2000, SMELTZER; BARE, 1998, OKUMA, 1998, RODRIGUES; DIOGO, 1996, BERGER; MAILLOUXPOIRIER, 1995, DUGAS, 1989) citam as diferentes modificações fisiológicas que ocorrem no idoso em relação ao aparelho músculo esquelético, incluindo ossos osteoporóticos, articulações aumentadas, tendões esclerosados, amplitude de movimento limitada, discos intervertebrais adelgaçados e fraqueza muscular.

Os ossos sofrem inúmeras alterações metabólicas, incluindo a supressão de estrogênio na menopausa, e a atividade reduzida contribui com a perda de massa óssea. Portanto, as mulheres perdem mais massa óssea do que os homens. Outras alterações poderiam ser citadas, como o desequilibrio da reabsorção de cálcio, a desmineralização constante da massa e da densidade óssea, o que se traduz numa maior porosidade e fragilidade do tecido ósseo além de encurtamento das vértebras dorso-lombares, pela osteoporose, produzindo um arqueamento da coluna vertebral (diminuição da estatura de 1,2 a 5 $\mathrm{cm}$ ); desvio da parte superior do tórax e acentuação da curva natural da coluna vertebral, e diminuição do volume da cavidade torácica, por osteoporose das costelas e diminuição da ação dos músculos respiratórios (SMELTZER; BARE, 1998).

O Fator Relacionado à "Força e resistência diminuidas" foi encontrado em $61,7 \%$ dos sujeitos. Segundo Okuma (1998), o envelhecimento associase, obrigatoriamente, à redução da capacidade aeróbia máxima, da força muscular, das respostas motoras mais eficientes, da capacidade funcional geral, ou seja, à redução da aptidão física. "O processo de envelhecimento do sistema muscular reduz tanto as forças estática e dinâmica máximas, quanto a potência e velocidade máximas" (p. 43). Smeltzer e Bare (1998) também afirmam que muitas vezes a mobilidade do individuo está restrita devido à perda da força muscular.

Sabemos que com o decorrer da idade, a elasticidade e estabilidade dos músculos, tendões e ligamentos se deterioram, a área transversal dos músculos torna-se menor pela atrofia muscular e a massa muscular diminui em proporção ao peso do corpo, o que leva a uma redução da força muscular. A diminuição da força é deteriorada cerca de $5 \%$ por década (OKUMA, 1998). Com essa redução, pode haver diferentes graus de comprometimento de habilidades motoras, já que, como afirma (OKUMA, 1998), toda e qualquer atividade a ser realizada necessita de um "limiar" de força mínima para a sua realização, ao passo que abaixo desse limiar o individuo se torna incapaz de manter sua independência e autonomia.

O Fator Relacionado ao "Enfraquecimento neuro-muscular" foi encontrado em $55 \%$ dos indivíduos. O sistema nervoso de pessoas idosas sofre algumas mudanças devido ao processo normal de envelhecimento e é extremamente vulnerável às doenças sistêmicas em geral. As fibras nervosas que se conectam diretamente com os músculos mostram um declínio de sua função, interferindo, por sua vez, nas funções neurológicas mais simples que envolvem um número de conexões na medula espinhal. Os idosos, freqüentemente, assumem uma postura fletida, apresentando rigidez muscular, tremores e movimentos lentos. Estas alterações neuromusculares prejudicam a motricidade e a coordenação da pessoa idosa, e são estas que se associam, dentre outras, com o aumento do risco de quedas e subseqüentes lesões, especialmente fraturas de quadril e fêmur (OKUMA, 1998, SMELTZER; BARE, 1998).

Trinta e dois individuos (53,3\%) apresentam "Dano perceptual ou cognitivo". Segundo Smeltzer; Bare (1998), com o avançar da idade ocorre uma diminuição da massa cerebral e, conseqüentemente, perdas no domínio cognitivo e perceptual do individuo. Okuma (1998) afirma que são as perdas do domínio cognitivo e as disfunções físicas que contribuem para a maior redução da independência do idoso, limitando suas possibilidades de viver confortável e satisfatoriamente, além de restringir sua atuação na sociedade.

"Dor ou desconforto" foi encontrada em 36,7\% dos idosos. Certos tecidos são mais sensiveis à dor que outros; por exemplo, o tecido muscular parece ser altamente sensivel a estimulos dolorosos e a dor de músculos contundidos ou isquêmicos pode ser lancinante. Por isso é necessário tomar muito cuidado ao se mover os pacientes com desordens que envolvam o sistema músculo esquelético. A dor piora, freqüentemente, durante às atividades fisicas. Em 
clientes com problemas músculo-esqueléticos, a dor, freqüentemente, está associada ao movimento da estrutura afetada. Quando um esforço físico ocasiona dores, pode-se ter de reduzir as atividades do cliente para impedir maiores lesões às células de seu organismo. No caso de dores fortes, eles, ocasionalmente, ficam rigidamente deitados, porque qualquer movimento intensifica o desconforto (BERGER; MAILLOUX-POIRIER, 1995; DUNGAS, 1989). Esta condição assume caráter especial para atenção aos idosos uma vez que estes têm menor senso da percepção dolorosa que os mais jovens. Uma vez que a clientela refere dor, devemos pensar em agentes causais em franca atividade.

"Intolerância à atividade física" foi identificada em $28,3 \%$ dos individuos. Okuma (1998) afirma que como uma conseqüência da diminuição da tolerância ao esforço físico, um grande número de pessoas idosas vivem abaixo do limiar da sua capacidade física, necessitando somente de uma mínima intercorrência na saúde para tornarem-se complemente dependentes. Segundo Berger e Mailloux-Poirier as intolerâncias à atividade, provem, muitas vezes, de uma falta de motivação para mover-se seguida de um período de imobilização prolongada ou uma patologia cardio-respiratória.

As modificações fisiológicas do sistema cardiovascular alteram significativamente a capacidade do idoso em realizar atividades físicas. Podemos citar como exemplo de algumas alterações da capacidade cardiovascular, a atrofia de células musculares cardiacas; diminuição da capacidade máxima do coração; diminuição da força das contrações cardiacas; aumento do consumo de oxigênio pelo coração; diminuição da elasticidade das artérias e arteríolas e diminuição do aporte sanguinea a todos os órgãos e glândulas por diminuição do débito cardiaco e perda de elasticidade das artérias. Ocorre uma diminuição da resistência física devido a redução da capacidade aeróbia máxima (OKUMA, 1998; SMELTZER; BARE, 1998).

O Fator Relacionado à "Depressão ou ansiedade severa" foi identificado em 15\% dos sujeitos. A depressão ou a ansiedade severa leva o individuo ao uso de medicamentos que podem provocar tremores, sonolência e prejudicar a mobilidade. As alterações de dimensão psicológicas são complexas e diferente de indivíduo para indivíduo. Berger e Mailloux-Poirier (1995) informam que a ansiedade e ou a depressão severas podem afetar a mobilidade do indivíduo, visto que uma pessoa muito ansiosa pode tornar-se hiperativa, não parando de movimentar-se, andar de uma lado para o outro ou ser incapaz de relaxar. Por outro lado, o individuo deprimido pode ficar "fadigado" e não se sentir motivado a realizar qualquer movimento.
As Características definidoras encontradas foram agrupadas, por entendermos que estas são identificadas conjuntamente, embora apareçam separadas na NANDA e no protocolo de avaliação por nós utilizado.

A inabilidade para mover-se significativamente dentro do ambiente físico foi observada em 83,63\% dos sujeitos (Tabela 2).

Autores como Smeltzer e Bare (1998), Kisner e Colby (1992) afirmam que cada articulação do corpo tem uma amplitude normal de movimento. Se esta amplitude é limitada, as funções da articulação e dos músculos que movem a articulação estão comprometidas, podendo desenvolver deformidades dolorosas, e impedir o individuo de realizar, independentemente, movimentos físicos no ambiente em que se encontra, incluindo o mover-se no leito (observada em $43,33 \%$ dos idosos investigados) ou levantar-se da cama (com uma freqüência de $40 \%$ dos sujeitos).

O uso de muletas, andador ou bengalas $(11,66 \%)$ aconteceu porque, muitas vezes, o individuo não consegue deambular independentemente, $e$ necessita da ajuda de outras pessoas e/ou de equipamentos (OKUMA, 1998).

Entre as diversas conseqüências do envelhecimento da estrutura óssea e articulações e do sistema muscular Morais, Braga e Silva (1995) citam: a diminuição da flexibilidade e o decréscimo do controle e rapidez dos movimentos, com alterações fisiológicas significantes. Soma-se a essas alterações a diminuição da flexibilidade (encontrado com 96,7\%), que segundo Meusel citado por Okuma, 1998, prejudica a resolução de problemas simples do cotidiano, como vestir-se, lavar-se, limpar a casa, entre outros.

A flexibilidade, definida por Okuma (1998), como a capacidade de movimento da articulação com a maior amplitude possivel, declina de $20 \%$ a $30 \%$ dos 20 aos 70 anos, com aumento nesse percentual depois dos 80 anos. Na velhice é comum a dificuldade em deambular independente. Em nosso estudo encontramos essa caracteristica em $53,33 \%$ dos sujeitos. Muitas vezes, como já referimos neste trabalho, o indivíduo necessita da ajuda de outras pessoas e/ou equipamentos como de cadeiras de rodas (identificado em $30 \%$ dos sujeitos).

Além disso, há estudos (PHILLIPS; HASKELL, citados por OKUMA, 1998) sobre quedas em idosos que associam a redução da força de membros inferiores (encontrada por nós em 53,33\%) à incapacidade em levantar-se de uma cadeira ou de uma cama (encontrada aqui com $40 \%$ de freqüência), com instabilidade ou pouco controle postural (apresentada por $43,33 \%$ dos sujeitos), com redução da amplitude da passada (encontrada $85,7 \% \mathrm{em}$ 
TABELA 2 - Distribuição das Características Definidoras para o Diagnóstico de Enfermagem Mobilidade Fisica Prejudicada em idosos no Abrigo Sagrada Família, Goiânia-2000

\begin{tabular}{|c|c|c|}
\hline Características Definidoras & $\mathrm{F}$ & $\%$ \\
\hline $\begin{array}{l}\text { Flexibilidade diminuida/ Diminuição da elasticidade/ } \\
\text { Redução da capacidade de movimentação/Diminuição da amplitude de } \\
\text { movimento. }\end{array}$ & 58 & 96,70 \\
\hline $\begin{array}{l}\text { Enrijecimento da articulação/ Comprometimento de articulações/ } \\
\text { Aumento da densidade e da rigidez da articulação. }\end{array}$ & 55 & 91,70 \\
\hline Estabilidade muscular comprometida/equilibrio. & 52 & 86,70 \\
\hline Amplitude da passada diminuida. ${ }^{(1)}$ & 36 & 85,70 \\
\hline Inabilidade para mover-se significativamente dentro do ambiente físico. & 46 & 83,63 \\
\hline Velocidade do andar reduzida ${ }^{(1)}$ & 34 & 80,90 \\
\hline Atrofia muscular/ Massa muscular dim inuída. & 47 & 78,30 \\
\hline Controle muscular diminuido/ Dificuldade na coord enação. & 47 & 78,30 \\
\hline Incapacidade para limpar a casa. & 45 & 75,00 \\
\hline Incapacidade para realizar o auto-cuidado. & 45 & 75,00 \\
\hline Tônus muscular diminuido. & 44 & 73,30 \\
\hline Incapacidade física para fazer compras. & 44 & 73,30 \\
\hline Incapacidade física para cozinhar. & 43 & 71,70 \\
\hline Incapacidade para utilizar transporte. & 42 & 70,00 \\
\hline Enfraquecimento muscular/ Força diminuida. & 41 & 68,30 \\
\hline Incapacidade física para lavar roupas. & 39 & 65,00 \\
\hline Incapacidade para banhar-se. & 38 & 63,33 \\
\hline Incapacidade para utilizar o vaso sanitário. & 38 & 63,33 \\
\hline Portador de deficiência física & 36 & 60,00 \\
\hline Redução da força dos membro superiores. & 33 & 55,00 \\
\hline Redução da força dos membro inferiores. & 32 & 53,33 \\
\hline Incapacidade para deambular independente e sem ajuda. & 33 & 53,33 \\
\hline Incapacidade para vestir-se. & 30 & 50,00 \\
\hline Incapacidade para levantar-se da cama. & 24 & 40,00 \\
\hline Inabilidade para mover-se significativamente no leito. & 26 & 43,30 \\
\hline Incapacidade para sentar-se em uma cadeira. & 26 & 43,30 \\
\hline Instabilidade ou pouco controle postural. & 26 & 43,30 \\
\hline Presença de contraturas musculares & 23 & 38,33 \\
\hline Restrição dos movimentos devido à dor. & 21 & 35,00 \\
\hline Restrito à cadeiras de rodas/acamados. & 18 & 30,00 \\
\hline Incapacidade para levar o alimento à boca. & 16 & 26,66 \\
\hline Uso de muletas, andador ou bengala & 07 & 11,66 \\
\hline Portador de paralisia. & 05 & 08,30 \\
\hline Restrição dos movimentos impostas por razões mecânicas. & 03 & 05,00 \\
\hline
\end{tabular}

(1) Dezoito individuos estão restritos à cadeira de rodas ou acamados. Esta característica não se aplica a estes individuos. Nesse caso $n=42$

nossa pesquisa) e da velocidade do andar (identificada em $80,9 \%$ da amostra). Também é muito comum no processo de envelhecimento a perda da velocidade do andar e da amplitude da marcha, que advêm da diminuição da força muscular (encontrada em nosso estudo com uma freqüência de $68,3 \%$ ), da massa (observada em $78,3 \%$ dos sujeitos) e da flexibilidade (encontrada em 96,7\%).

Uma das principais limitações na amplitude do movimento articular com a idade resulta do aumento da proporção do tecido conjuntivo na massa muscular, da desidratação da articulação da mudança de sua composição em colágeno e elastina. Este conjunto de alterações leva ao aumento da densidade e da rigidez da articulação (identificada em $91,7 \%$ dos idosos da instituição), levando à diminuição da amplitude de movimento (OKUMA, 1998).

O modo com a pessoa anda é avaliado quando ela caminha um curta distância perto do examinador, que observa o passo, sua suavidade e ritmo. 0 movimento articular limitado e uma variedade de condições neurológicas podem afetar a marcha (SMELTZER; BARE, 1998).

Morais, Braga e Silva (1995) afirmam que o idoso está mais susceptivel a perda do equilibrio (observada em 86,7\% da amostra) devido às alterações nos órgãos do sentido, pois estes informam o indivíduo sobre a posição e a orientação, e à medida que estes se tornam menos sensiveis, maior a fragilidade do individuo. 
Além de ocorrer a diminuição progressiva de metade do peso total dos músculos entre 30 e os 70 anos, há uma perda crescente no número de fibras musculares devido à atrofia da miofibrila com substituição do tecido fibroso, ocasionando perda da massa muscular (SMELTZER; BARE, 1998). A hipotrofia muscular foi encontrada em nossa pesquisa em $78,3 \%$ dos sujeitos.

A perda da capacidade motora, segundo Okuma (1998), leva à incapacidade para realizar as Atividades da Vida Diária (AVDs) e as Atividades Instrumentais da Vida Diária (AIVDs). As primeiras referem-se às atividades de cuidados pessoais básicos e as segundas referem-se a tarefas mais complexas do cotidiano e incluem, necessariamente, aspectos do uma vida independente. Os sujeitos apresentaram alteração em AVDs como vestir-se (50\%), banhar-se $(63,3 \%)$, levantar-se da cama (40\%) e sentar-se numa cadeira $(43,3 \%)$, utilizar o banheiro/vaso sanitário $(63,3 \%)$, comer/levar alimento à boca $(26,66 \%)$. Além destas, foram identificadas alterações em AIVDs, como fazer compras $(73,33 \%)$, cozinhar $(71,7 \%)$, limpar a casa $(75 \%)$, lavar roupa $(65 \%)$, utilizar meios de transporte $(70 \%)$.

A Diminuição da Força Muscular foi encontrada em $68,33 \%$ dos idosos e Dificuldade de Coordenação foi identificada em $78,33 \%$ dos sujeitos. Segundo Okuma (1998), estes fenômenos decorrem de efeitos funcionais resultantes das alterações musculares fisiológicas próprias do envelhecimento. Tais efeitos contribuem para uma diminuição da capacidade em realizar atividades diárias e elevam o risco de incapacitação física.

É bem conhecido o fato de que, com o decorrer da idade, a elasticidade e estabilidade dos músculos tornam-se menores. Este fenômeno foi apresentado por $96,7 \%$ dos avaliados. Estes processos decorrem, por sua vez, da atrofia e diminuição da massa muscular, que foram identificadas em $78,33 \%$ dos idosos da instituição pesquisada. Além disso, a perda de elasticidade muscular aliada perda de elasticidade articular a leva a um prejuizo na flexibilidade, identificada em $96,7 \%$ do grupo estudado. As degenerações/enrijecimento articulares foram encontradas em $91,7 \%$ dos sujeitos.

Em nosso instrumento de coleta de dados, a Caracteristica Definidora "Portador de deficiência fisica" foi observada em dois itens, "Portador de deficiência neuropsicomotora" e "Portador de deformidade músculo-esquética", que apareceram respectivamente em $25,45 \%$ e $45,45 \%$ dos sujeitos avaliados. Para identificamos a Característica Definidora "Portador de deficiência física", consideramos os indivíduos que apresentam pelo menos um dos itens acima descritos, o que aconteceu com 36 individuos, perfazendo $60 \%$ da amostra.
Smeltzer e Bare (1998) afirmam que os problemas mais comuns associados à imobilidade incluem músculos enfraquecidos (identificada em $68,33 \%$ da população estudada), contraturas das articulações (encontrada por nós em $38,33 \%$ dos sujeitos) e deformidades músculo esqueléticas (observada em $45,45 \%$ dos idosos).

Alguns dos sinais que indicam a probabilidade de a mobilidade da pessoa estar restrita são a dor $(35 \%)$, a paralisia $(08,3 \%)$, a perda da força muscular $(68,33 \%)$, a presença de um aparelho de imobilização ( por exemplo, gesso, tipóia_ 05\%).

Dugas (1989) afirma que as contraturas dos músculos (observada em $38,18 \%$ dos sujeitos) e o enrijecimento das articulações (encontrada em 91,7\% dos idosos avaliados) são efeitos da inatividade prolongada.

No idoso ocorre um redução fisiológica da capacidade motora que pode ser patologicamente induzida ou agravada. A degeneração da capacidade motora pode ser acelerada na ausência de intervenções adequadas, o que deve ser objeto de atenção tanto na população idosa da comunidade, como da institucionalizada.

Segundo os autores Smeltzer e Bare (1998), Morais, Braga e Silva (1995), a prevenção ou a prática de exercicios podem evitar agravamentos que ocorrem no aparelho motor com o avançar da idade. Por isso acreditamos que seja importante a elaboração de intervenções de Enfermagem.

As intervenções de cuidados terapêuticos para sujeito com alterações de mobilidade, principalmente no idoso, visam a eliminação dos efeitos prejudiciais da imobilidade, maximizando a mobilidade de acordo os limites de cada individuo (Berger; Mailloux-Poirier, 1995). Nesse sentido, cabem intervenções de Enfermagem em conjunto à ações de todos os membros da equipe de saúde

\section{CONCLUSÃO}

Este estudo buscou identificar a ocorrência de Mobilidade Fisica Prejudicada em Idosos institucionalizados, seus fatores relacionados e características definidoras. Participaram 60 idosos com idade de 60 a 105 anos, moradores de um asilo filantrópico da cidade de Goiânia. Verificamos o índice de ocorrência deste diagnóstico igual a $100 \%$ dos sujeitos.

Os Fatores Relacionados de maior prevalência foram: Enfraquecimento Músculo-esquelético e Força e Resistência Diminuidas, que foram identificados em mais de $60 \%$ dos idosos.

Quanto às Caracteristicas Definidoras, encontramos como maior freqüentes (E $80 \%$ ): diminuição da capacidade de movimentos/diminuição 
da amplitude / diminuição flexibilidade/ diminuição elasticidade; capacidade diminuida de mover significativamente no ambiente; comprometimento articular/ aumento da densidade e rigidez da articulação; equilibrio/ estabilidade muscular comprometida; amplitude da passada diminuida e Velocidade do andar diminuída.

Num segundo nivel de ocorrência (entre 50 a $79,9 \%$ ), temos Caracteristicas Definidoras como: diminuição da massa muscular/ atrofia muscular; capacidade física diminuida para limpar casa; capacidade física diminuida para compras; capacidade física diminuida para vaso sanitário; capacidade física diminuida para vestir-se, entre outras.

O Enfermeiro deve estar preparado para identificar precocemente o Diagnóstico de Enfermagem Mobilidade Fisica Prejudicada no idoso, e desenvolver intervenções numa perspectiva de atuação multiprofissional, frente a alterações na mobilidade do individuo, ajudando-o a manter ou evitar prejuizos nesta área.

\section{REFERÊNCIAS BIBLIOGRÁFICAS}

ARAÚJO, L.A. de O.; BACHION,M.M. Protocolo de Validação de Conteúdo do Diagnóstico de Enfermagem "Mobilidade Física Prejudicada" em idosos. Trabalho apresentado no 10. Seminário Nacional de Pesquisa em Enfermagem, Gramado/RS, 24-27 de maio de 1999.

BACHION, M. M. et al. Validação de conteúdo do Diagnóstico de Enfermagem "Mobilidade Física Prejudicada" em idosos. Trabalho apresentado no 51. Congresso Brasileiro de Enfermagem e 10. Congresso Panamericano de Enfermeria, Florianópolis, 1999.

BERGER, L.M; MAILLOUX-POIRIER, M. Pessoas idosas: uma abordagem global. Lisboa: Lusodidacta, 1995.
CARPENITO, L. J. Diagnóstico de Enfermagem: aplicação à prática clínica. 6. ed. Porto Alegre: Artes Médicas, 1997

DOENGES, M. E.; MOORHOUSE, M.F. Diagnóstico e Intervenção em enfermagem. Tradução Regina Machado Garcez. 5. ed. Porto Alegre: ARTMED, 1999

DUGAS, B. W. Enfermagem Prática. 4. ed. Rio de Janeiro: Guanabara , 1994.

KISNER, C.; COLBY, L.A. Exercícios terapêuticos: fundamentos e técnicas. 2. ed. São Paulo: Manole, 1992

MORAIS, I. B. de; BRAGA, T. G.; SILVA, V. T. S. da. Princípios básicos de Enfermagem em geriatria: ações preventivas. 2. ed. Brasilia: Gráfica Valci Editora, 1995.

NORTH AMERICAN NURSING DIAGNOSIS ASSOCIATION. Diagnósticos de Enfermagem da NANDA. Definições e classificação 1999-2000. (Trad. Jeanne Liliane M. Michel). Porto Alegre: ARTMED, 2000.

NORTH AMERICAN NURSING DIAGNOSIS ASSOCIATION. Nursing diagnosis: definitions \& classification 1995-1996. Philadelphia/EUA: NANDA, 1994.

OKUMA, S. S. O idoso e a atividade Física: fundamentos e pesquisa. Campinas: Papirus, 1998. Coleção Vivaidade.

PAPALÉO NETTO, M. Gerontologia. A velhice, o envelhecimento em visão globalizada. São Paulo: Atheneu, 2000.

RODRIGUES, R. A. P.; DIOGO, M.J. Como cuidar dos idosos. São Paulo: Papirus, 1996.

SMELTZER, S. C.; BARE, B.G. Tratado de Enfermagem Médico-Cirúrgia. 8. ed. Rio de Janeiro: Guanabara Koogan, 1998. 